\title{
Sinonasal mucosal melanoma: a 10-year experience of 36 cases in China
}

\author{
Tan Wang ${ }^{1,2,3}$, Yue Huang ${ }^{1,2,3 \#}$, Jingrong Lu $^{1,2,3}$, Mingliang Xiang ${ }^{2,3,4}$ \\ ${ }^{1}$ Department of Otorhinolaryngology, Head and Neck Surgery, Xinhua Hospital, Shanghai Jiaotong University School of Medicine, Shanghai, \\ China; ${ }^{2}$ Ear Institute, Shanghai Jiaotong University School of Medicine, Shanghai, China; ${ }^{3}$ Shanghai Key Laboratory of Translational Medicine \\ on Ear and Nose diseases, Shanghai, China; ${ }^{4}$ Department of Otorhinolaryngology, Head and Neck Surgery, Ruijin Hospital, Shanghai Jiaotong \\ University School of Medicine, Shanghai, China \\ Contributions: (I) Conception and design: M Xiang; (II) Administrative support: M Xiang, J Lu; (III) Provision of study materials or patients: M \\ Xiang, T Wang; (IV) Collection and assembly of data: T Wang; (V) Data analysis and interpretation: T Wang, Y Huang; (VI) Manuscript writing: \\ All authors; (VII) Final approval of manuscript: All authors. \\ \#These authors contributed equally to this work. \\ Correspondence to: Mingliang Xiang. Department of Otorhinolaryngology, Head and Neck Surgery, Ruijin Hospital Affiliated to Shanghai Jiao \\ Tong University School of Medicine, 197 Ruijin $2^{\text {nd }}$ Road, Shanghai, China. Email: mingliangxiang@163.com; Jingrong Lu. Department of \\ Otorhinolaryngology, Head and Neck Surgery, Xinhua Hospital Affiliated to Shanghai Jiao Tong University School of Medicine, 1665 Kongjiang \\ Road, Shanghai, China; Email: lvjingrong@xinhuamed.com.cn.
}

Background: Sinonasal mucosal melanoma (SNMM) is a rare tumor with a poor prognosis. This study aimed to assess the clinical and imaging features, progression, treatment, and possible prognostic factors of SNMM.

Methods: Thirty-six patients with SNMM were retrospectively reviewed in the Department of Otolaryngology \& Head and Neck Surgery of Xinhua Hospital from January 2008 to December 2017.

Results: The age of the first diagnosis was $67.4 \pm 10.8$ years; the most common clinical symptoms included epistaxis, nasal obstruction, headache, and facial pain. Most tumors originated in the nasal cavity (63.9\%) and at stage IV (77.8\%). Melanin in melanoma showed typical signal intensity on magnetic resonance imaging (MRI), T1WI had high signal while T2WI had low signal. 41.6\% of patients had the typical MRI findings. Treatment included surgery, surgery with radiotherapy, and radiotherapy only. The follow-up time ranged from 4 to 96 months, with a median time of 22 months, 1-, 3-, and 5-year OS is $80.6 \%, 36.1 \%$, and $13.9 \%$, respectively. The 3 -year OS was better in cases in the $\mathrm{T} 3$ stage than the $\mathrm{T} 4$ stage $(\mathrm{P}=0.02)$. However, tumors that originated from the paranasal sinus had a poorer prognosis than the nasal cavity $(\mathrm{P}=0.04)$. The cases receiving postoperative radiotherapy showed poorer prognosis $(\mathrm{P}=0.02)$. Other factors were not found to be associated with prognosis, including gender, age, lymph node metastasis, distant metastasis, computed tomography (CT) enhancement, and typical MRI findings.

Conclusions: The SNMM was a devastating tumor with poor outcomes; most cases were diagnosed at late stages, which may account for poor prognosis. Tumors with melanin feature MRI findings do not have a better prognosis. The treatment of postoperative radiotherapy is still controversial.

Keywords: Nose neoplasm; malignant melanoma, imaging, prognosis

Submitted Jul 10, 2020. Accepted for publication Aug 10, 2020.

doi: $10.21037 / \mathrm{atm}-20-5575$

View this article at: http://dx.doi.org/10.21037/atm-20-5575 


\section{Introduction}

Sinonasal mucosal melanoma (SNMM) is a rare tumor, accounting for less than $1.0 \%$ of all malignant melanoma. However, it accounts for more than $72.0 \%$ of all malignant melanoma in the head and neck. SNMM had few early specific symptoms, which tended to miss diagnosis; patients showed epistaxis and nasal obstruction at an early stage when the tumors invaded into the tissues surrounding the nasal cavity and sinus, the patients may have diplopia, headache, hyposmia and facial pain (1). The results of computed tomography (CT) and magnetic resonance imaging (MRI) may be beneficial to the diagnosis and the assessment of SNMM, the CT may indicate the size, location, and extent of the tumor and with or without cervical lymph node metastasis. Characteristic MRI findings were found in some patients, including highsignal on T1WI and low signal on T2WI, but some patients lacked typical MRI signals due to poor tumor differentiation or intra-tumoral hemorrhage (2). However, the relationship between SNMM images and clinical features is still unclear.

There are many treatments for SNMM, including surgery, radiotherapy, chemotherapy, and biological therapy. Among them, surgery is the primary treatment, complete surgical resection is essential to improve the prognosis, however, because the complex anatomical structure of the nasal cavity and sinus, tumors are usually closed to some critical structures, like the base of the skull and eye socket. Therefore, complete surgical resection is not simple; some advanced patients even lose the opportunity for surgery. Instead, they only received palliative radiotherapy and chemotherapy (3). The incidence of SNMM is increasing year by year, but its prognosis is still poor despite advances in the field of therapy of malignant melanoma. The 5-year overall survival (OS) rate of cutaneous malignant melanoma is about 70.0 $80.0 \%$. However, SNMM has worse outcomes with an OS rate of less than $30.0 \%$, which shows high invasive, easily recurrence, and distant metastasis.

In this study, we reported our experience of treating SNMM over the past ten years, to assess the clinical and imaging features, progression, treatment, and possible prognostic factors. We present the following article in accordance with the STROBE reporting checklist (available at http://dx.doi.org/10.21037/atm-20-5575).

\section{Methods}

\section{Patients}

Thirty-six patients were retrospectively collected from the Department of Otolaryngology \& Head and Neck Surgery, Xinhua Hospital, Shanghai Jiao Tong University School of Medicine, from January 2008 to December 2017, all of them were diagnosed SNMM according to the histopathological results. The exclusion criterion was that patients without CT enhanced scan or enhanced MRI before the operation. The patients whose primary tumors were not found in the nasal cavity and sinus were excluded. Their treatments were discussed in a multidisciplinary team meeting with otolaryngologists, oncologists, and radiation therapists. This work was conducted in accordance with the Declaration of Helsinki (as revised in 2013) and was approved by ethics committee of Xinhua Hospital. The requirement for informed consent was waived because of the retrospective nature of the research.

\section{Data collection}

Epidemiological data, imaging results, treatment, tumor histopathology, and prognosis were obtained by chart review. The TNM stages were analyzed according to the $7^{\text {th }}$ edition of the American Joint Committee on Cancer (AJCC) classification (4). The typical signal intensity of MRI was defined as a high signal on T1WI and low signal on T2WI.

\section{Data analysis}

The results were expressed as means \pm standard deviation (SD) analyzed with SPSS v.20.0. The OS was defined as the time from first treatment to death from any cause. Clinical prognostic factors were compared using Fisher's exact test and the Mann-Whitney U test. The Kaplan-Meier method estimated survival, and any difference in survival was evaluated with a stratified log-rank test. A probability $\mathrm{P}<0.05$ was considered a significant difference.

\section{Results}

\section{Patient demographics and clinical symptoms}

Thirty-six patients with SNMM were included in this study, with an age of $67.4 \pm 10.8$ years and a male-female 
Table 1 Patient demographics, clinical symptoms, tumor location, TNM stage, and treatment

\begin{tabular}{|c|c|}
\hline Characteristic & $\mathrm{N}=36$ \\
\hline Age (years): mean \pm SD & $67.4 \pm 10.8$ \\
\hline \multicolumn{2}{|l|}{ Gender } \\
\hline Female & $17(47.2 \%)$ \\
\hline Male & $19(52.8 \%)$ \\
\hline \multicolumn{2}{|l|}{ Clinical symptoms } \\
\hline Epistaxis & $26(72.2 \%)$ \\
\hline Nasal obstruction & $30(83.3 \%)$ \\
\hline Facial pain & $10(27.8 \%)$ \\
\hline \multicolumn{2}{|l|}{ Origin } \\
\hline Nasal cavity (inferior turbinate/others) & $23(14 / 9)(63.9 \%)$ \\
\hline Maxillary sinus & $10(27.8 \%)$ \\
\hline Ethmoidal sinus & $3(8.3 \%)$ \\
\hline \multicolumn{2}{|l|}{ AJCC T stage } \\
\hline T3 & $8(22.2 \%)$ \\
\hline $\mathrm{T} 4 \mathrm{a}$ & $23(63.9 \%)$ \\
\hline $\mathrm{T} 4 \mathrm{~b}$ & $5(13.9 \%)$ \\
\hline \multicolumn{2}{|l|}{ AJCC N stage } \\
\hline No & 27 (75.0\%) \\
\hline N1 & $9(25.0 \%)$ \\
\hline \multicolumn{2}{|l|}{ AJCC M stage } \\
\hline MO & $32(88.9 \%)$ \\
\hline M1 & $4(11.1 \%)$ \\
\hline \multicolumn{2}{|l|}{ TNM stage } \\
\hline Stage III & $8(22.2 \%)$ \\
\hline Stage IVa & $20(55.6 \%)$ \\
\hline Stage IVb & $4(11.1 \%)$ \\
\hline Stage IVc & $4(11.1 \%)$ \\
\hline \multicolumn{2}{|l|}{ Treatment } \\
\hline Surgery only & $20(55.6 \%)$ \\
\hline Surgery + radiotherapy & $13(36.1 \%)$ \\
\hline Radiotherapy only & 3 (8.3\%) \\
\hline
\end{tabular}

Table 2 The relationship between tumor location and TNM staging

\begin{tabular}{lcccc}
\hline Tumor primary site & Stage III & Stage IVa & Stage IVb & Stage IVc \\
\hline Nasal cavity & 8 & 14 & 0 & 1 \\
Maxillary sinus & 0 & 6 & 3 & 1 \\
Ethmoidal sinus & 0 & 0 & 1 & 2 \\
\hline
\end{tabular}

ratio of 1:1.12. The mean interval between first signs and diagnosis was $10 \pm 3.2$ months. Among 36 patients, epistaxis, nasal obstruction, headache, and facial pain were the most common clinical symptoms, present in $72.2 \%, 83.3 \%$, and $27.8 \%$ patients, respectively (Table 1 ).

\section{Tumor location and TNM stage}

As first diagnosed, $63.9 \%$ of all tumors were originated in the nasal cavity, $27.8 \%$ in the maxillary sinus, $8.3 \%$ in the ethmoidal sinus. According to the $7^{\text {th }}$ edition of the AJCC classification, $22.2 \%$ of patients were staged in T3, $63.9 \%$ in T4a, $13.9 \%$ in $\mathrm{T} 4 \mathrm{~b}$, lymph node metastasis was found in 9 cases, and distant metastasis was found in 4 cases (one liver, one lung and two brains). AJCC TNM stage for SNMM defined T3 as malignant melanoma that limited to mucosa with no invasion into deeper tissue. Patients in T3 stage with no evidence for lymph node or distant metastasis were classified in stage III according to the clinical staging regulation. The absence of stage I and II indicated the highly malignancy of the SNMM. In a word, $22.2 \%$ of patients were staged in stage III, $55.6 \%$ in stage IVa, $11.1 \%$ in stage IVb, $11.1 \%$ in stage IVc (Table 1). Moreover, all the patients with tumors in the nasal cavity were at stage III, and IVa, most maxillary sinus, and ethmoidal sinus were at stage IVb and IVc (62.5\%) (Table 2).

\section{Imaging results}

CT and MRI were used to predict the margin of tumor invasion. The CT findings revealed that $61.1 \%$ (22 cases) of patients had bone destruction, and $69.4 \%$ of tumors showed enhancement in varying scales. It was reported that melanoma showed typical signal intensity on MRI. T1WI was a high signal, while T2WI was a low signal (Figure 1). However, only $41.6 \%$ of patients had the typical MRI 

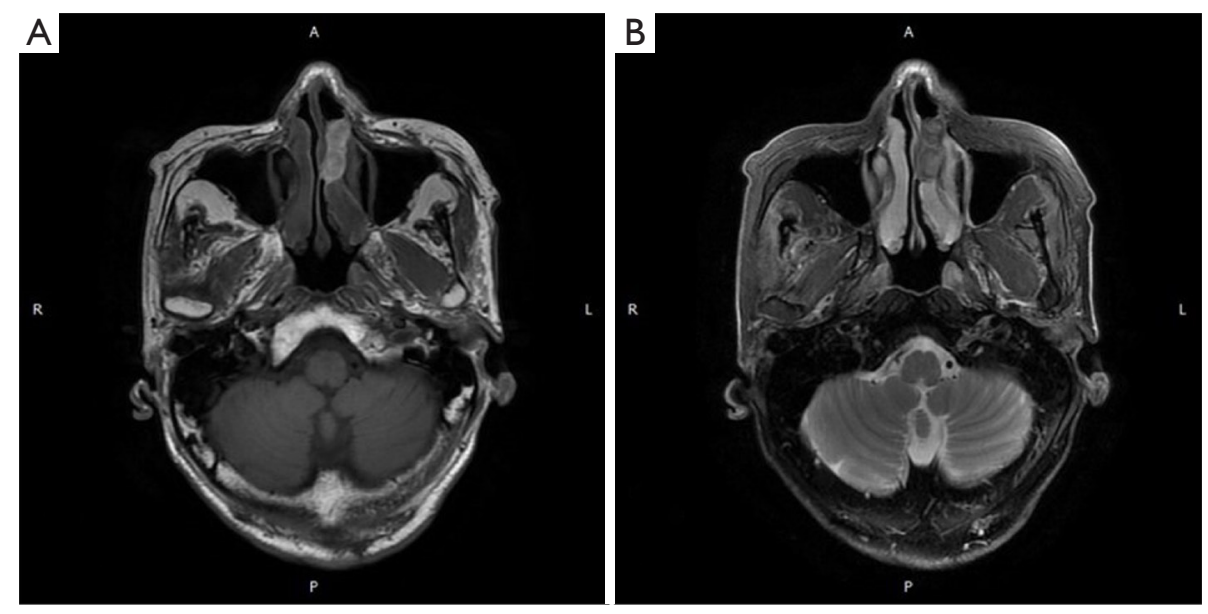

Figure 1 A male patient aged 80 years presented with the typical MRI findings. (A) High signal on T1WI; (B) low signal on T2WI. MRI, magnetic resonance imaging.

findings. The rest of the patients presented various MRI features, including mixed signals in T1WI and T2WI, low signals in T1WI, and high signals in T2WI, the equal signal in T1WI and high signal in T2WI, etc.

\section{Treatment}

Thirty-three cases underwent surgery; among them, 30 cases had an endoscopic approach; one case underwent a lateral rhinotomy operation, one case had a Caldwell-Luc approach operation, one case had endoscopic combined with the Caldwell-Luc approach. Thirteen cases underwent postoperative adjuvant radiotherapy, and the other 3 cases had radiotherapy only through distant metastasis (Table 1).

\section{Follow-up and prognosis}

The follow-up time ranged from 4 to 96 months, with a median time of 22 months. During follow-up, ten patients $(27.78 \%)$ relapsed, four patients, showed locoregional lymph node involvement, and three patients showed distant metastasis, including parotid gland, lung, and liver. The 1 -year OS was $80.6 \%$, the 3 -year OS was $36.1 \%$, and the 5-year OS was $13.9 \%$.

\section{Factors predicting $O S$}

As shown in Table 3, no significant differences of OS were found in gender ( 1 -year OS $\mathrm{P}=0.68$; 3 -year OS $\mathrm{P}=0.73$; 5 -year OS $\mathrm{P}=0.65)$ and age $(1$-year $\mathrm{P}=0.68,3$-year $\mathrm{P}=007$;
5 -year $\mathrm{P}=1.00)$. The 3 -year OS was better in cases in the T3 stage than the $\mathrm{T} 4$ stage $(\mathrm{P}=0.02)$. The lymph-node metastasis and distant metastasis did not affect OS. Tumors originating from the paranasal sinus had a poorer prognosis than the nasal cavity (Figure $2 A, \mathrm{P}=0.04$ ). In our study, the CT enhancement degree did not influence prognosis (Figure $2 B, \mathrm{P}=0.12$ ). The cases with typical MRI findings, high signal on T1WI, and low signal on T2WI, did not show a better prognosis (Figure $2 C, \mathrm{P}=0.49$ ). Interestingly, adjuvant radiation therapy did not improve the OS. On the contrary, the cases that received postoperative radiotherapy showed poorer prognosis (Figure 2D, $\mathrm{P}=0.02$ ). The recurrence rate for those who received radiotherapy had no significant difference between those who had surgery only (38.46\% and $25.00 \%, \mathrm{P}=0.26$ ).

\section{Discussion}

SNMM is a devastating tumor. Our results showed that the 5 -year OS of SNMM was 13.9\%, which was consistent with the findings of other studies. Thompson et al. (5) pointed out that SNMM patients older than 60 years had a worse prognosis, Jangard et al. (6) pointed out that males and the patients older than 60 years had higher mortality rates. However, our results were opposite, which were like the study of Konuthula et al. (7), which concluded that gender and age did not affect the prognosis. In our study, the SNMM patients older than 80 years were found to be prone to frequent relapses. However, they were usually long-time survivors, even distant metastasis occurred. This may be 
Table 3 Clinical prognostic factors

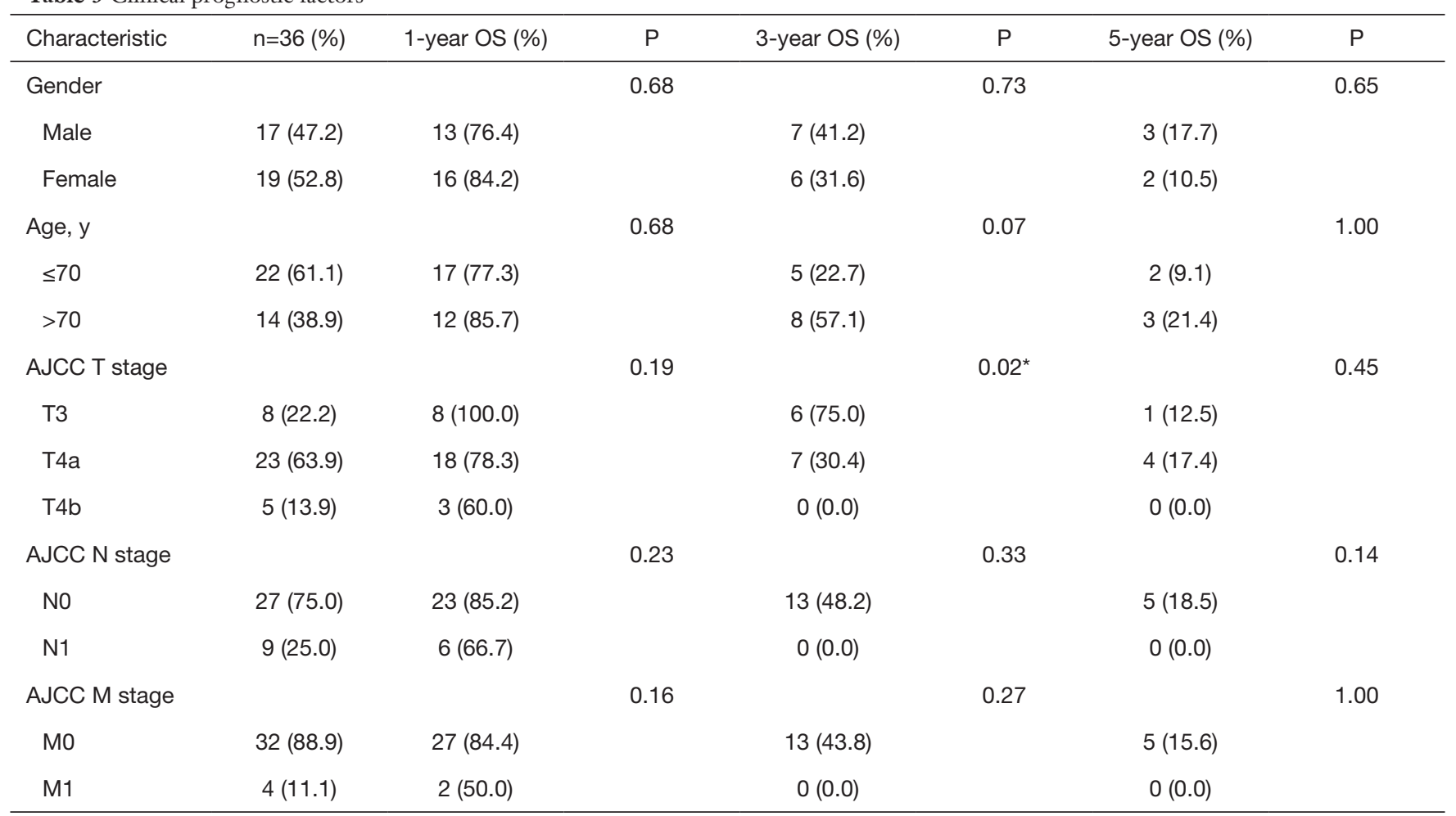

*, $\mathrm{P}<0.05$.

related to the different behavior of different subtypes.

The nasal mucosa was the most common site of SNMM (8), in the present study, the patient was at earlier TNM stage when SNMM site was located in the nasal cavity, besides, we found that 3-year OS was better in cases in T3 stage than in T4 stage $(\mathrm{P}=0.02)$, our results were like the other studies. But 1- and 5-year survival rate had no significant difference in our study. The lack of large number of cases may led to the result. Khan et al. found that patients with tumors confined to the nasal cavity had a better prognosis than patients with tumors involving sinus (9). Another study showed that the most common site of the tumor was a nasal wall (51.5\%), followed by the paranasal sinus $(19.1 \%)$ when SNMM was located in the nasal wall or septum, the 3- and 5-year OS was more significant than that in the entire nasal cavity (10). We found that tumors were originated from the nasal cavity, and the inferior turbinate had a better prognosis $(\mathrm{P}=0.04)$. Since clinical symptoms like nasal congestion and epistaxis were earlier observed in the cases with tumors found in the nasal mucosa. On the contrary, few symptoms were observed at an early stage if tumors were in the sinus, most patients were not diagnosed until the tumors invaded into surrounding tissues. Therefore, they lost the opportunity for radical surgery at the initial stages, leading to a worse prognosis.

In our study, lymph node metastasis was found in $25.0 \%$ of cases. The findings of Maldonado-Mendoza et al. (11) indicated that $10.0-20.0 \%$ of cases had lymph node metastasis at the first diagnosis; another $20.0 \%$ of cases had lymph node metastasis as the disease progressed. Amit et al. (12) believed that more than 89.0\% of SNMM patients did not have lymph node metastasis, risk factors of prognosis included surgical margin positive, skull invasion, and distant metastasis; however, lymph node metastasis was not the risk factor. Our results suggested that lymph node metastasis was not an essential factor in affecting the OS as well. In sum, prophylactic cervical lymph node dissection is not recommended in SNMM patients.

Image findings may show some biological characteristics of tumors. Kim et al. found that $87.1 \%$ of SNMM cases showed a high T1WI signal, which was significantly higher than $20.8 \%$ of other tumors (13). A study suggested that the MRI signal was associated with melanin in the tumor, the more melanin the tumor contained, the higher the signal on 

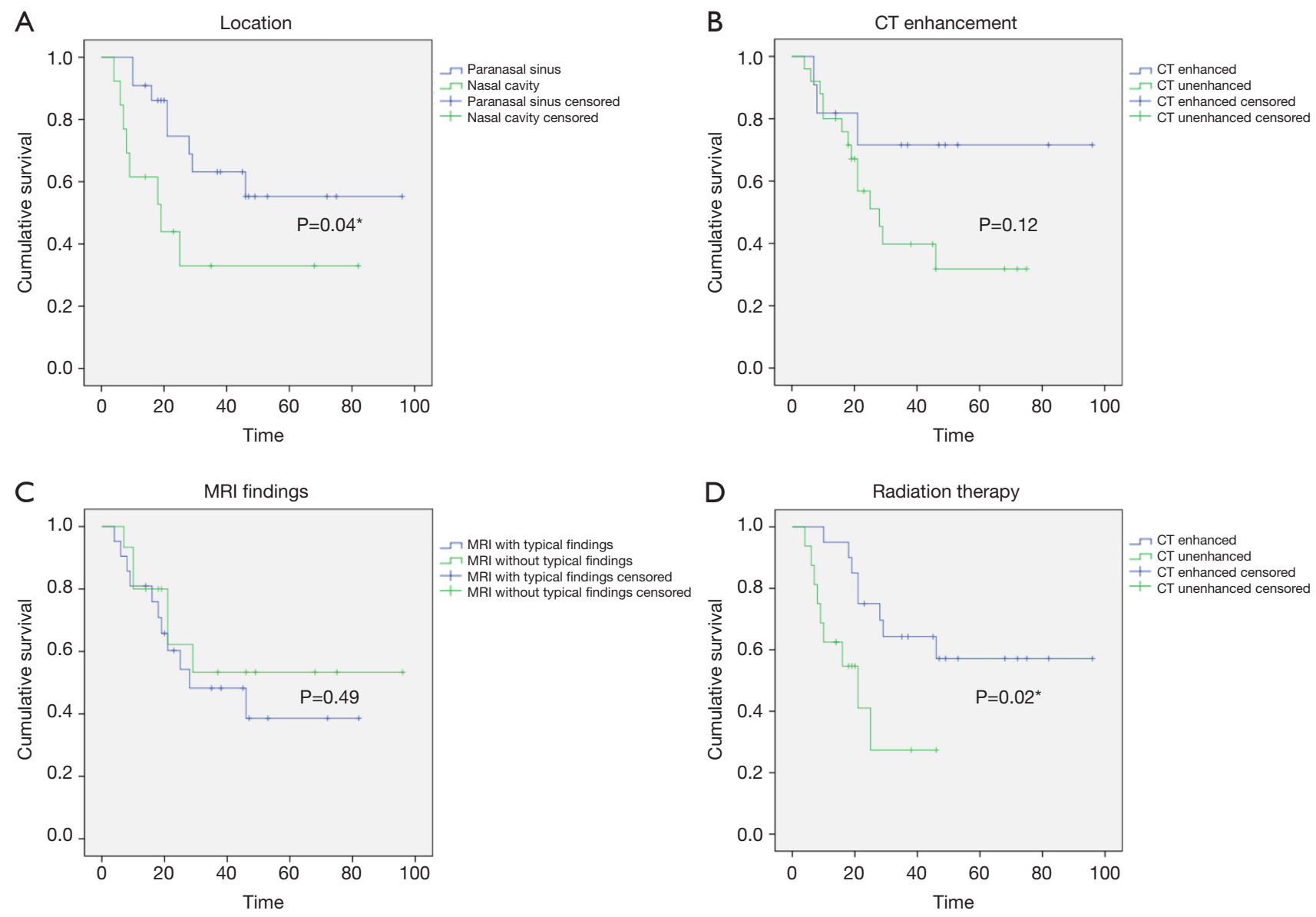

Figure 2 Kaplan-Meiler survival curve of SNMM. (A) Survival curve of SNMM located in the paranasal sinus and nasal cavity. (B) Survival curve of SNMM with and without CT enhancement. (C) Survival curve of SNMM with and without typical MRI findings. (D) Survival curve of SNMM with and without radiation therapy. *, P<0.05. CT, computed tomography; MRI, magnetic resonance imaging; SNMM, sinonasal mucosal melanoma.

T1WI and the lower signal on T2WI it showed in MRI (14). In our study, typical melanin MRI findings were observed in $41.6 \%$ of cases. They did not show a better prognosis than the cases without typical melanin MRI findings (Figure $2 C, \mathrm{P}=0.49$ ). According to the imaging theory of MRI, melanin pigment could be detected with typical imaging features. We believed that the SNMM could be divided into different subtypes based on MRI findings. In further research, we would compare the histopathological result with the imaging features of the tumor. Investigate the potential value of imaging examination for prognosis prediction. Although typical MRI findings show no clinical value in predicting prognosis, it may have a particular value in the differential diagnosis of tumors in the nasal or sinus.

Surgery is the preferred treatment of SNMM, including traditional open surgery and endoscopic surgery. Several studies showed that no difference of marginnegative resection rate, OS, and DFS was found between open surgery and endoscopic surgery. Moreover, no difference was found between two groups of postoperative complications like cerebrospinal fluid leakage and bleeding, as well as local recurrence and distant metastasis (15-18). In this study, 30 cases accepted endoscopic surgery. While 3 cases accepted open surgery, all of them were at late stages like IVa and IVb, which contributed to poor prognosis. In our study, 1-year OS was $80.6 \%$, 3-year OS was $36.1 \%$, and 5 -year OS was $13.9 \%$, like other reported studies. The results in the present study showed that endoscopic surgery was valuable, for it may leave light surgical trauma than open surgery while obtaining similar therapeutic 
efficacy. In sum, compared to open surgery, we consider that endoscopic surgery is valuable to be recommended for SNMM patients at the initial stages.

Radiotherapy is a standard adjuvant treatment for malignant tumors of the nasal cavity and sinus, which can effectively control local recurrence. A meta-analysis showed that postoperative radiotherapy might reduce the probability of local recurrence; however, it did not improve the survival rate (19). Christopherson et al. found that compared with radiotherapy alone, postoperative radiotherapy had higher DFS and OS rates, and lower recurrence rate (20). Another study compared the treatment effects of surgery with that of surgery combined radiotherapy, there was no significant difference of the 5-year OS between the two groups, but the recurrence rate was significantly different, the 5-year local recurrence rates of surgery and surgery combined radiotherapy were $55.6 \%$ and $29.9 \%$, respectively (12). Interestingly, our results indicated that surgery combined radiotherapy did not improve the recurrence rate; on the contrary, our results indicated higher prognosis in the patients with only surgery. Our results were different from earlier studies; it may be due to many causes. Some studies suggested SNMM was not sensitive to radiotherapy. Moreover, in our study, the patients with radiotherapy were mostly in stage IV, whose tumors were not completely removed surgically. In sum, the efficacy of postoperative radiotherapy for SNMM is still controversial, which needs further study.

\section{Conclusions}

All 36 cases in this study were diagnosed at stage III and IV. Although a better 3-year OS was found in cases in the T3 stage than the T4 stage, the prognosis of SNMM was still poor. Forty-one point six percent of patients had the typical MRI findings; however, no clinical value in predicting prognosis was found. Endoscopic surgery was valuable to be recommended to SNMM patients. Prophylactic cervical lymph node dissection was unhelpful, and the efficacy of postoperative radiotherapy for SNMM was still controversial.

\section{Acknowledgments}

Funding: None.

\section{Footnote}

Reporting Checklist: The authors have completed the STROBE reporting checklist. Available at http://dx.doi. org/10.21037/atm-20-5575

Data Sharing Statement: Available at http://dx.doi. org/10.21037/atm-20-5575

Conflicts of Interest: All authors have completed the ICMJE uniform disclosure form (available at http://dx.doi. org/10.21037/atm-20-5575). The authors have no conflicts of interest to declare.

Ethical Statement: The authors are accountable for all aspects of the work in ensuring that questions related to the accuracy or integrity of any part of the work are appropriately investigated and resolved. This work was conducted in accordance with the Declaration of Helsinki (as revised in 2013) and was approved by ethics committee of Xinhua Hospital. The requirement for informed consent was waived because of the retrospective nature of the research.

Open Access Statement: This is an Open Access article distributed in accordance with the Creative Commons Attribution-NonCommercial-NoDerivs 4.0 International License (CC BY-NC-ND 4.0), which permits the noncommercial replication and distribution of the article with the strict proviso that no changes or edits are made and the original work is properly cited (including links to both the formal publication through the relevant DOI and the license). See: https://creativecommons.org/licenses/by-nc-nd/4.0/.

\section{References}

1. Tajudeen BA, Vorasubin N, Sanaiha Y, et al. Sinonasal mucosal melanoma: 20-year experience at a tertiary referral center. Int Forum Allergy Rhinol 2014;4:592-7.

2. Yentz S, Lao CD. Immunotherapy for mucosal melanoma. Ann Transl Med 2019;7:S118.

3. Robin TP, Jones BL, Gordon OM, et al. A comprehensive comparative analysis of treatment modalities for sinonasal malignancies. Cancer 2017;123:3040-9.

4. Edge SB, Compton CC. The American Joint Committee on Cancer: the 7th edition of the AJCC cancer staging manual and the future of TNM. Ann Surg Oncol 2010;17:1471-4.

5. Thompson LD, Wieneke JA, Miettinen M. Sinonasal tract and nasopharyngeal melanomas: a clinicopathologic study of 115 cases with a proposed staging system. Am J Surg Pathol 2003;27:594-611. 


\section{Page 8 of 8}

6. Jangard M, Hansson J, Ragnarsson-Olding B. Primary sinonasal malignant melanoma: a nationwide study of the Swedish population, 1960-2000. Rhinology 2013;51:22-30.

7. Konuthula N, Khan MN, Parasher A, et al. The presentation and outcomes of mucosal melanoma in 695 patients. Int Forum Allergy Rhinol 2017;7:99-105.

8. Khademi B, Moradi A, Hoseini S, et al. Malignant neoplasms of the sinonasal tract: report of 71 patients and literature review and analysis. Oral Maxillofac Surg 2009;13:191-9.

9. Khan MN, Kanumuri VV, Raikundalia MD, et al. Sinonasal melanoma: survival and prognostic implications based on site of involvement. Int Forum Allergy Rhinol 2014;4:151-5.

10. Sun CZ, Li QL, Hu ZD, et al. Treatment and prognosis in sinonasal mucosal melanoma: A retrospective analysis of 65 patients from a single cancer center. Head Neck 2014;36:675-81.

11. Maldonado-Mendoza J, Ramirez-Amador V, AnayaSaavedra G, et al. Clinicopathological characterization of primary oral and sinonasal melanoma in a referral centre in Mexico City: 2000-2012. Int J Oral Maxillofac Surg 2015;44:427-32.

12. Amit M, Tam S, Abdelmeguid AS, et al. Approaches to regional lymph node metastasis in patients with head and neck mucosal melanoma. Cancer 2018;124:514-20.

13. Kim YK, Choi JW, Kim HJ, et al. Melanoma of the Sinonasal Tract: Value of a Septate Pattern on Precontrast T1-Weighted MR Imaging. AJNR Am J Neuroradiol

Cite this article as: Wang T, Huang Y, Lu J, Xiang M. Sinonasal mucosal melanoma: a 10-year experience of 36 cases in China. Ann Transl Med 2020;8(16):1022. doi: 10.21037/atm-205575
Wang et al. Prognostic factors of the sinonasal mucosal melanoma

2018;39:762-7.

14. Geninatti Crich S, Alberti D, Szabo I, et al. MRI visualization of melanoma cells by targeting overexpressed sialic acid with a $\mathrm{Gd}(\mathrm{III})$-dota-en-pba imaging reporter. Angew Chem Int Ed Engl 2013;52:1161-4.

15. Lund VJ, Chisholm EJ, Howard DJ, et al. Sinonasal malignant melanoma: an analysis of 115 cases assessing outcomes of surgery, postoperative radiotherapy and endoscopic resection. Rhinology 2012;50:203-10.

16. Swegal W, Koyfman S, Scharpf J, et al. Endoscopic and open surgical approaches to locally advanced sinonasal melanoma: comparing the therapeutic benefits. JAMA Otolaryngol Head Neck Surg 2014;140:840-5.

17. Breik O, Sim F, Wong T, et al. Survival Outcomes of Mucosal Melanoma in the Head and Neck: Case Series and Review of Current Treatment Guidelines. J Oral Maxillofac Surg 2016;74:1859-71.

18. Lundberg M, Haapaniemi A, Hagstrom J, et al. Similar survival outcome after endoscopic and open approaches for sinonasal mucosal melanoma. Rhinology 2019;57:132-8.

19. Li W, Yu Y, Wang H, et al. Evaluation of the prognostic impact of postoperative adjuvant radiotherapy on head and neck mucosal melanoma: a meta-analysis. BMC Cancer 2015;15:758.

20. Christopherson K, Malyapa RS, Werning JW, et al. Radiation therapy for mucosal melanoma of the head and neck. Am J Clin Oncol 2015;38:87-9.

(English Language Editor: J. Chapnick) 